\title{
IgM multiple myeloma with an extremely rare non-aggressive presentation: A case report
}

\author{
THOMAS GREUTER ${ }^{1}$, MARTIN BROWNE ${ }^{2}$, CORINA DOMMANN-SCHERRER ${ }^{3}$, \\ DANIEL BINDER $^{3}$, CHRISTOPH RENNER ${ }^{4}$ and URSULA KAPP ${ }^{1}$
}

\author{
${ }^{1}$ Department of Medicine, GZO-Zurich Regional Health Center, 8620 Wetzikon, Zurich, Switzerland; \\ ${ }^{2}$ North Coast Cancer Institute, Coffs Harbour Health Campus, Coffs Harbour, New South Wales 2450, Australia; \\ ${ }^{3}$ Division of Hematology, Cantonal Hospital Winterthur, 8401 Winterthur; \\ ${ }^{4}$ Oncology Centre, Hirslanden Clinic, 8032 Zurich, Switzerland
}

Received March 4, 2015; Accepted June 16, 2016

DOI: $10.3892 / \mathrm{ol} .2016 .5034$

\begin{abstract}
In the present study, the case of a 41-year-old man with immunoglobulin (Ig)M multiple myeloma (MM) that presented with an unusually non-aggressive clinical course who has survived for $>9$ years to date, is presented. Initial diagnosis of symptomatic MM was established according to the International Myeloma Working Group consensus statement and guidelines. Due to the mild symptoms, no therapy was administered and the patient was closely followed up. Eight years after initial diagnosis, clinical, morphological and genetic progression occurred with the development of hypercalcemia, progressively deteriorating polyneuropathy, clonal expansion of plasma cells up to $50 \%$ of hematopoietic cells and demonstration of the typical $t(11 ; 14)$ translocation (Ig heavy chain locus rearrangement). Subsequently, 4 cycles of induction chemotherapy with velcade, cyclophosphamide and dexamethasone, were administered. At the time of writing, the patient remained alive in generally good health. To the best of our knowledge, with a survival time of $>9$ years, this case reports the longest survival time of an IgM MM patient to date, which contradicts previous evidence that suggests IgM MM exhibits an aggressive clinical course.
\end{abstract}

\section{Introduction}

Multiple myeloma (MM) accounts for $10 \%$ of all hematological malignancies and is a B-cell malignancy characterized by a clonal proliferation of abnormal plasma cells in the bone marrow $(1,2)$. MM occurs most frequently in patients between the ages of 50 and 80 years (3). MM is characterized by the presence of monoclonal immunoglobulin (Ig) in the serum, of which

Correspondence to: $\mathrm{Dr}$ Thomas Greuter, Department of Medicine, GZO-Zurich Regional Health Center, Spitalstrasse 66, 8620 Wetzikon, Zurich, Switzerland

E-mail: th_greuter@bluewin.ch

Key words: IgM, multiple myeloma, bone marrow biopsy, immunohistochemistry
$\operatorname{IgG}$ and $\operatorname{Ig} \mathrm{A}$ are the most frequently observed subtypes (4). $\operatorname{Ig} \mathrm{M}$ $\mathrm{MM}$ is an extremely rare lymphoproliferative disease associated with an aggressive clinical course, which accounts for $<0.5 \%$ of all MM cases $(4,5)$. Distinguishing IgM MM from Waldenström's macroglobulinemia (WM) is extremely important, but is often challenging due to similar characteristics (6). Differentiation may be established based on clinical presentation or bone marrow morphology (7). Diagnosis of IgM MM is usually established based on the International Myeloma Working Group criteria, which state that IgM MM includes: i) The presence of M-protein in the serum and/or urine; ii) $>10 \%$ bone marrow clonal plasma cells or plasmacytoma; and iii) associated organ or tissue impairment (end organ damage, including bone lesions) (8). The median reported survival for IgM MM is 36 months (9). In this study, the case of a 41-year-old man who presented with immunoglobulin (Ig)M multiple myeloma (MM) with an unusually non-aggressive clinical course is presented. A watch-and-wait strategy was adopted, however clinical progression occurred after eight years. At present the patient remains alive nine years after the initial diagnosis. Written informed consent was obtained from the patient for publication of this case report and any accompanying images.

\section{Case report}

In October 2005, a 41-year-old man with an unremarkable medical history presented to the Coffs Harbour Health Campus, Coffs Harbour, Australia, with the complaint of tingling and burning paresthesia in both feet and exertional dyspnea. No hepatosplenomegaly or lymphadenopathy was identified and physical examination was otherwise unremarkable. The laboratory examination results at initial presentation were as follows: White blood cell count, $6.2 \times 10^{9} / 1$ (reference range, 4.0-12.0x10 $/ 1$ ); hemoglobin, $150 \mathrm{~g} / 1$ (reference range, 130-180 g/1); platelet count, $281 \times 10^{9} / 1$ (reference range, $\left.150-400 \times 10^{9} / 1\right)$; erythrocyte sedimentation rate, $115 \mathrm{~mm} / \mathrm{h}$ (reference range, $1-10 \mathrm{~mm} / \mathrm{h}$ ); creatinine, $0.10 \mathrm{mmol} / 1$ (reference range, 0.05-0.12 mmol/l), blood urea nitrogen, $6.7 \mathrm{mmol} / 1$ (reference range, 2.5-6.5 mmol/l); calcium, $2.61 \mathrm{mmol} / \mathrm{l}$ (reference range, 2.22-2.65 mmol/l); lactate dehydrogenase, $171 \mathrm{IU} / 1$ (reference range, 60-200 IU/1). All other routine laboratory 
parameters tested were within the normal ranges. Serum electrophoresis revealed a homogeneous peak in the gamma fraction, which was identified as IgM kappa by immunofixation. Nephelometry identified an elevated concentration of $\operatorname{IgM}$ (49.00 g/l; reference range, 0.44-2.76 g/l) and reduced $\operatorname{IgA}$ $(0.25 \mathrm{~g} / \mathrm{l}$; reference range, $0.80-4.12 \mathrm{~g} / \mathrm{l})$ and $\mathrm{IgG}(3.89 \mathrm{~g} / \mathrm{l}$; reference range, $6.45-13.90 \mathrm{~g} / \mathrm{l})$ concentrations. $\beta_{2}$-microglobulin levels were $1.42 \mathrm{mg} / \mathrm{l}$ (reference range, $0-20 \mathrm{mg} / \mathrm{l}$ ). Magnetic resonance imaging of the spine revealed no evidence of lytic bone lesions. Consecutive bone marrow (BM) aspirate morphology revealed moderate diffuse infiltration with $12 \%$ plasma cells. A small number of plasma cells exhibited atypical multinucleated forms ( $\leq 3$ nuclei) or cytoplasmic vacuolation. BM biopsy demonstrated diffuse proliferation of plasma cells expressing cluster of differentiation (CD)38 and IgM exhibiting kappa restriction. Lymphoplasmocytic lymphoma/WM was excluded by morphology and immunohistochemistry. No cytogenetic abnormalities were identified. Diagnosis of symptomatic IgM kappa MM was established based on the presence of an M-protein in the serum, plasma cell infiltration in the BM and associated tissue impairment (polyneuropathy), according to the International Myeloma Working Group consensus statement and guidelines (8). Due to the mild clinical presentation and mild end organ damage observed, a watch-and-wait strategy was selected. During the following 8 years, the patient exhibited an extremely benign course of the disease. However, as a result of continuously increasing IgM levels and progressive polyneuropathy, BM aspirate/biopsy was repeated in November 2012 and March 2014. Giemsa staining, which was performed at room temperature overnight, was used for morphological analysis. Immunohistochemical analyses were performed using standard techniques and antibodies to CD3, CD4, CD5, CD8, CD10, CD19, CD20, CD56, CD38, CD45 and CD138. Morphology demonstrated diffuse infiltration with $10 \%$ abnormal plasma cells, which were positive for IgM kappa (Figs. 1 and 2). The immunophenotype observed was consistent with the presence of plasma cells with abnormal cells exhibiting positivity for CD38, CD138, CD10 and negativity for CD19 and CD56, as demonstrated by flow cytometric analysis. In addition, interphase fluorescence in situ hybridization analysis was performed using samples enriched for CD138-positive plasma cells. The results revealed a translocation involving myeloma overexpressed (11q13) and immunoglobulin heavy locus (IGH) (14q32), and therefore positivity for $\mathrm{t}(11 ; 14)$ (q13;q32), an additional signal for IGH (14q32), loss of one copy of MAF bZIP transcription factor (16q23), deleted in lymphocytic leukemia 1 (13q14) and fibroblast growth factor receptor 4 (4p16). In May 2014, elevated calcium levels $(2.75 \mathrm{mmol} / \mathrm{l}$; reference range, $2.1-2.55 \mathrm{mmol} / \mathrm{l}$ ), deteriorating polyneuropathy and the identification of IGH locus rearrangement resulted in the initiation of chemotherapy treatment. The patient was administered 4 cycles of induction chemotherapy: velcade $\left(1.3 \mathrm{~g} / \mathrm{m}^{2}\right.$ subcutaneously; days 1, 4, 8 and 11), cyclophosphamide $\left(500 \mathrm{mg} / \mathrm{m}^{2}\right.$ intravenously; days 1 and 8 ) and dexamethasone (40 mg; days 1 , $2,4,5,8,9,11$ and 12 ) with cycle 2 starting at day 22 , cycle 3 at day 43 and cycle 4 at day 64 . No evidence of lytic bone lesions was identified on whole body bone computed tomography. In July 2014, the patient had completed his last cycle of induction chemotherapy with VCD. At the time of writing this manuscript (December 2014), the patient remains in good health and the

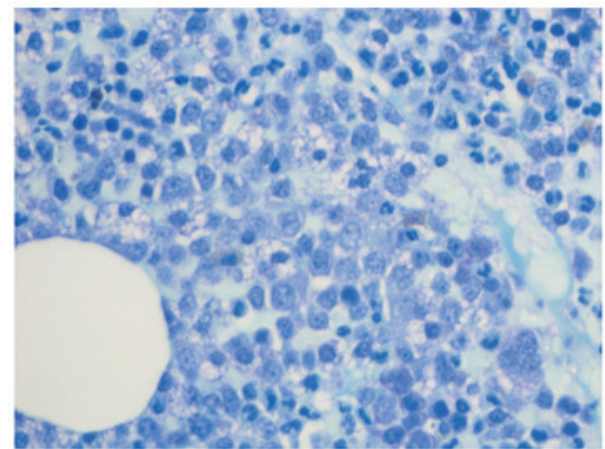

Figure 1. Bone marrow biopsy demonstrating proliferation of atypical enlarged plasma cells (stain, Giemsa; magnification x640).

A

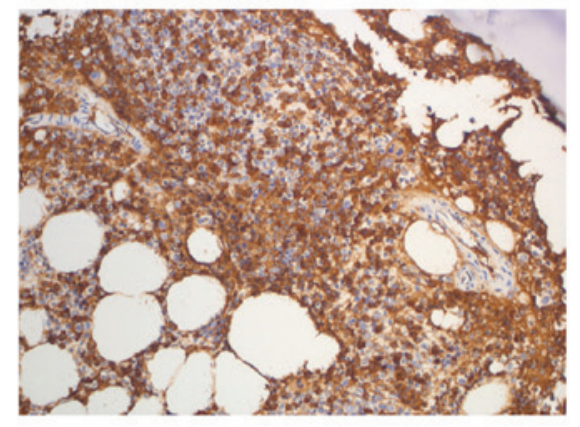

B
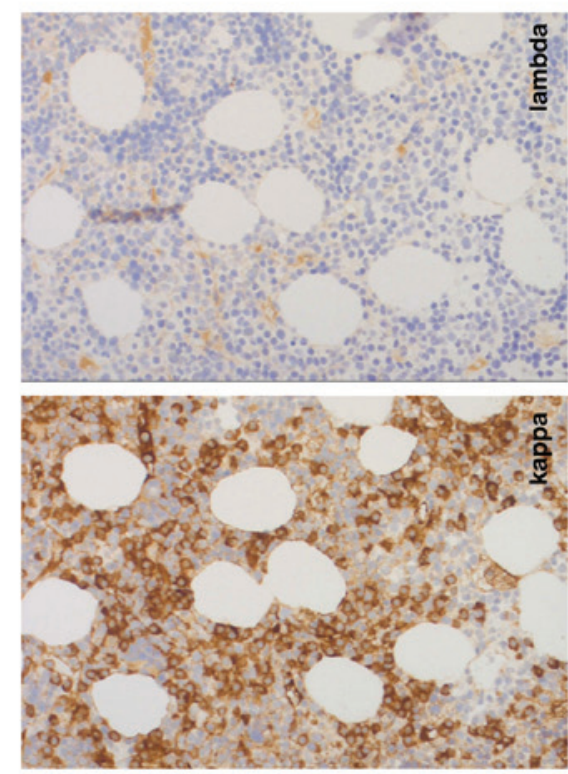

C

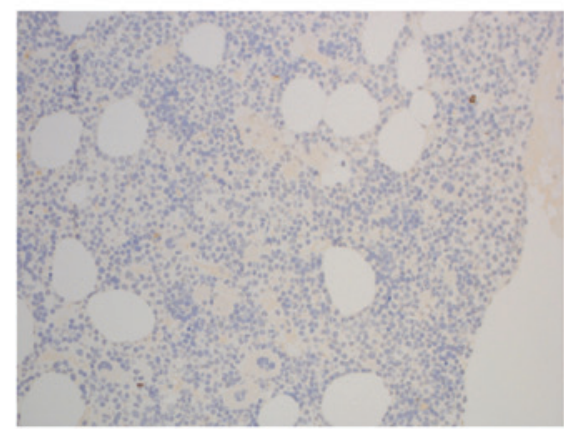

Figure 2. Immunohistochemical analysis of the bone marrow biopsy. (A) Neoplastic plasma cells containing monoclonal cytoplasmic immunoglobulin $\mathrm{M}$ with (B) light chain restriction kappa and negativity for lambda. (C) Neoplastic plasma cells exhibiting negative staining for cluster of differentiation 20. Magnification, x200. 
symptoms of polyneuropathy have improved slightly following initiation of chemotherapy.

\section{Discussion}

Distinguishing IgM MM from WM is critical; however, it may be difficult. IgM MM and WM are two distinct hematological entities with the common symptom of an $\operatorname{IgM}$ monoclonal gammopathy (6). Differentiation may be established based on clinical presentation or BM morphology. The clinical symptoms of anemia, hypercalcemia, renal impairment, lytic bone lesions, and plasma cell infiltration of BM clearly indicate the rare diagnosis of IgM MM (7). However, the above-mentioned criteria are not highly sensitive. Lymphadenopathy and hepatosplenomegaly, two symptoms of WM, are typically present in only 20-40\% of all WM cases (6). Furthermore, bone lesions are not always present in IgM MM (10). Recently, the presence of $\mathrm{t}(11 ; 14)$ in IgG MM has been demonstrated to be highly specific (11). Translocation $\mathrm{t}(11 ; 14)$ leads to dysregulation of cyclin D1 and has been identified in 7/8 patients with IgM myeloma in a study by Avet-Loiseau et al (11), while no such translocation was identified in all 17 cases of WM. In addition, recently a mutation in exon 5 of the MYD88 gene (MYD88 L265P), which is absent in IgM MM patients, was demonstrated to be specific for WM with a specificity of $>90 \%$ (12). Due to the extremely rare incidence of $\operatorname{IgM} \mathrm{MM}$, only a few case series have been reported in the literature to date $(4,5,9)$. Notably, IgM MM appears to be more aggressive than IgG or IgA MM, as well as WM, with an overall median survival time of 36 months (9). In addition, IgM MM has a poor clinical outcome in the context of high-dose therapy (13). To date, the longest reported survival time of a patient with $\operatorname{IgM}$ myeloma was 60 months (5). At present, treatment guidelines recommend the same therapeutic approach for IgM MM and IgG or IgA myelomas (14). Notably, IgM monoclonal gammopathy of unknown significance has a benign course with no progression to $\operatorname{IgM}$ MM (15).

In the present case, the clinical presentation was atypical; the patient initially presented with polyneuropathy, without hypercalcemia, anemia, renal impairment or lytic bone lesions. However, diagnosis of symptomatic MM was established according to the International Myeloma Working Group consensus statement and guidelines (8). Due to the mild symptoms observed, no therapy was initiated and the patient was closely followed up. However, 8 years after initial diagnosis clinical, morphological and genetic progression occurred, with the development of hypercalcemia, progressively deteriorating polyneuropathy, clonal expansion of plasma cells up to 50 of hematopoietic cells and demonstration of the typical $t(11 ; 14)$ translocation (IGH locus rearrangement). In conclusion, to the best of our knowledge, the survival time of the symptomatic IgM MM patient in this case is the longest to be reported in the literature to date, which contradicts previous evidence that suggests IgM MM exhibits an aggressive clinical course (9).
IgM MM may have a more heterogeneous disease course than was previously understood. However, larger observational studies are required in order to identify the potential risk and protecting factors that result in an aggressive or comparatively non-aggressive $\operatorname{IgM}$ MM course, respectively.

\section{References}

1. Kyle RA and Rajkumar SV: Multiple myeloma. Blood 111: 2962-2972, 2008.

2. Kyle RA: Diagnostic criteria of multiple myeloma. Hematol Oncol Clin North Am 6: 347-358, 1992.

3. Boccadoro M and Pileri A: Diagnosis, prognosis, and standard treatment of multiple myeloma. Hematol Oncol Clin North Am 11: 111-131, 1997.

4. De Gramont A, Grosbois B, Michaux JL, Peny AM, Pollet JP, Smadja N, Krulik M, Debray J, Bernard JF and Monconduit M: IgM myeloma: 6 cases and a review of the literature. Rev Med Interne 11: 13-18, 1990 (In French).

5. Dierlamm T, Laack E, Dierlamm J, Fiedler W and Hossfeld DK: IgM myeloma: A report of four cases. Ann Hematol 81: 136-139, 2002.

6. Dimopoulos MA, Panayiotidis P, Moulopoulos LA, Sfikakis P and Dalakas M: Waldenström's macroglobulinemia: Clinical features, complications, and management. J Clin Oncol 18: 214-226, 2000.

7. Schuster SR, Rajkumar SV, Dispenzieri A, Morice W, Aspitia AM, Ansell S, Kyle R and Mikhael J: IgM multiple myeloma: Disease definition, prognosis, and differentiation from Waldenstrom's macroglobulinemia. Am J Hematol 85: 853-855, 2010.

8. Dimopoulos M, Terpos E, Comenzo RL, Tosi P, Beksac M, Sezer O, Siegel D, Lokhorst H, Kumar S, Rajkumar SV, et al; IMWG: International myeloma working group consensus statement and guidelines regarding the current role of imaging techniques in the diagnosis and monitoring of multiple myeloma. Leukemia 23: 1545-1556, 2009.

9. Annibali O, Petrucci MT, Del Bianco P, Gallucci C, Levi A, Foà R and Avvisati G: IgM multiple myeloma: Report of four cases and review of the literature. Leuk Lymphoma 47: 1565-1569, 2006.

10. Haghighi B, Yanagihara R and Cornbleet PJ: IgM myeloma: Case report with immunophenotypic profile. Am J Hematol 59: 302-308, 1998.

11. Avet-Loiseau H, Garand R, Lodé L, Harousseau JL and Bataille R; Intergroupe Francophone du Myélome: Translocation $\mathrm{t}(11 ; 14)(\mathrm{q} 13 ; \mathrm{q} 32)$ is the hallmark of $\operatorname{IgM}$, IgE, and nonsecretory multiple myeloma variants. Blood 101: 1570-1571, 2003.

12. Xu L, Hunter ZR, Yang G, Zhou Y, Cao Y, Liu X, Morra E, Trojani A, Greco A, Arcaini L, et al: MYD88 L265P in Waldenström macroglobulinemia, immunoglobulin $\mathrm{M}$ monoclonal gammopathy, and other B-cell lymphoproliferative disorders using conventional and quantitative allele-specific polymerase chain reaction. Blood 121: 2051-2058, 2013.

13. Morris C, Drake M, Apperley J, Iacobelli S, van Biezen A, Bjorkstrand B, Goldschmidt H, Harousseau JL, Morgan G, de Witte T, et al; Myeloma Subcommittee of Chronic Leukaemia Working Party of EBMT: Efficacy and outcome of autologous transplantation in rare myelomas. Haematologica 95: 2126-2133, 2010.

14. Moreau P, San Miguel J, Ludwig H, Schouten H, Mohty M, Dimopoulos M and Dreyling M; ESMO Guidelines Working Group: Multiple myeloma: ESMO Clinical Practice Guidelines for diagnosis, treatment and follow-up. Ann Oncol 24 (Suppl 6): vi133-vi137, 2013.

15. Kyle RA, Therneau TM, Rajkumar SV, Remstein ED, Offord JR, Larson DR, Plevak MF and Melton LJ III: Long-term follow-up of IgM monoclonal gammopathy of undetermined significance. Blood 102: 3759-3764, 2003. 